\title{
LA PALEONTOLOGÍA ESPAÑOLA HOY. PERSPECTIVAS DE FUTURO
}

\author{
Pascual RIVAS CARRERA \\ Departamento de Estratigrafía y Paleontología. Universidad de Granada. Granada 18071.
}

\begin{abstract}
Pascual Rivas Carrera 1996. La paleontologia española hoy. Perspectivas de futuro. [The spanish paleontology actually. Futu-
\end{abstract} re perspective]. Revista Española de Paleontología, N" Extraordinario, 9-20. ISSN 0213-6937.

\section{INTRODUCCIÓN}

El "hoy" es un acercamiento intelectual a un proceso dinámico, por ello, pensando que interesa conocer la dinámica de nuestra ciencia, se ha tomado el "hoy" como el fin de una secuencia de unos diez años. La evolución de la Paleontología no tiene un discurrir continuo y las previsiones para el futuro inmediato pierden en parte su valor cuando se aplican a medio y largo plazo; por ello habrá que hacerlas, no sobre los intereses inmediatos, sino enmarcando la Paleontología española en los contextos científico y social, nacional e internacional. Consecuentemente en este trabajo subyacen dos enfoques:

a) Relación entre el próximo pasado y la actualidad.

b) Análisis de la actualidad y demandas de futuro.

El primero tendrá más peso en el estudio de las cuestiones más formales, el segundo en el de las substanciales. El análisis de la situación actual se basará en:

a) El acervo paleontológico español y en la consulta directa a algunos miembros de su comunidad.

b) En el vaciado desde 1985, o desde su fundación, de una serie de publicaciones (Tabla 1). Se han escogido estas porque permiten conocer la producción de mayor difusión dentro de España y la incidencia de los paleontólogos españoles en la comunidad internacional, así como el interés de esa comunidad por las publicaciones españolas. Esta claro que lo analizado no agota lo publicado y es difícil estudiar el total y su relación con los datos que se contienen en este trabajo. Basándose en los de Linares (1993), la relación puede ser de cuatro a uno. Para las publicaciones de mayor difusión (SCI) la relación, en cambio, es de dos a uno.

c) A la información anterior hay que añadir la obtenida en trabajos de intenciones similares a este: Molina (1982), Vera (1987), Aguirre (1989), Truyols (1988), Babin (1992), Ramírez del Pozo (1992), Linares (1993), etc., al tiempo ha sido de gran ayuda lo contenido en los tratados de Briggs \& Crowther (1991) y López Martínez \& Truyols (1994), o los de congresos y reuniones (Martinell, ed., 1981; Álvarez \& Conway Morris, eds., 1992).

\section{PALEONTÓLOGOS}

Se han contabilizado treinta y cinco centros: universidades, organismos públicos de investigación (OPI), museos, empresas, etc., que tienen paleontólogos de plantilla o activos, considerando como tales a los que han publicado algún trabajo (naturalmente en las revistas consultadas) desde 1985. (Tabla 1) El mayor número corresponde a departamentos universitarios; los centros en empresas se han obtenido de los datos de Ramírez del Pozo (1992). Por no estar aún

\begin{tabular}{|c|c|c|}
\hline $\begin{array}{l}\text { GEOLOGIA } \\
\text { ESPAÑA }\end{array}$ & $\begin{array}{c}\text { PALEONTOLOGIA } \\
\text { ESPAÑA }\end{array}$ & $\begin{array}{c}\text { PALEONTOLOGIA } \\
\text { INTERNACIONALES SCI }\end{array}$ \\
\hline $\begin{array}{c}\text { Estudios geológicos } \\
\text { Rev. Soc. Geol. España } \\
\text { Geogaceta }\end{array}$ & $\begin{array}{c}\text { Rev. Esp. Paleontologia } \\
\text { Jornadas Paleontologia } \\
\text { B. R. Soc. Esp. Hist. Nat } \\
\text { Rev. Esp. Micropaleontologia }\end{array}$ & $\begin{array}{c}\text { Paleobiology } \\
\text { Palaeontology } \\
\text { J. Paleontology } \\
\text { Palaeo } 3 \\
\text { Geobios } \\
\text { Lethaia } \\
\text { Micropaleontology }\end{array}$ \\
\hline
\end{tabular}

Tabla 1. Publicaciones consultadas. Las españolas se han escogido con la idea de recoger al mayor número de autores.

definidos administrativamente o estar sólo al inicio de sus actividades, sin plantilla definida, no se han incluido el Museo de Sevilla-La Cartuja, el Departamento de Geología de la Universidad de Cádiz, el Museo de Orce y el Departamento de Biología Animal de la Universidad de La Laguna.

Hay quince centros más que hace diez años, de estos algunos son nuevos, otros se corresponden a la entrada de paleontólogos en departamentos preexistentes y otros con la vuelta a la actividad paleontológica de investigadores dedi-

\begin{tabular}{|c|c|c|c|c|c|}
\hline \multicolumn{7}{|c|}{ DISTRIBUCION DE CENTROS } \\
\hline \multirow{2}{*}{ UNIVERSIDAD } & C. S. I. C. & $\begin{array}{c}\text { Centros } \\
\text { Mixtos } \\
\text { UNIV-CSIC }\end{array}$ & $\begin{array}{c}\text { PUBLICOS } \\
\text { regionales } \\
\text { y locales }\end{array}$ & $\begin{array}{c}\text { Otros } \\
\text { Centros } \\
\text { Públicos }\end{array}$ & EMPRESAS \\
\hline 21 & 3 & 2 & 5 & 2 & $2(+2)$ \\
\hline $60 \%$ & $8,6 \%$ & $5,7 \%$ & $14.3 \%$ & $5,7 \%$ & $5,7 \%$ \\
\hline
\end{tabular}

Tabla 2. Centros con paleontólogos. En el caso de empresas no se contabilizan "Otros centros públicos" que tienen configuración legal de empresas públicas. 
cados a otros temas durante años. Sólo un 5,7\% de los investigadores de plantilla pertenece a empresas, lo que contrasta con lo datos de otros países (Morales y Rivas, 1986). Dentro de la iniciativa pública son muy numerosos los centros de la Universidad, y hay que destacar, por el esfuerzo personal y económico que ha supuesto su puesta en marcha y mantenimiento, los de carácter cultural fundados por entes regionales y locales, un $20 \%$ del total (Tabla 2).

La plantilla de paleontólogos (tablas 3 y 4 ) la componen 127 funcionarios y contratados permanentes de entes públicos y privados; aunque los últimos alcanzan sólo un

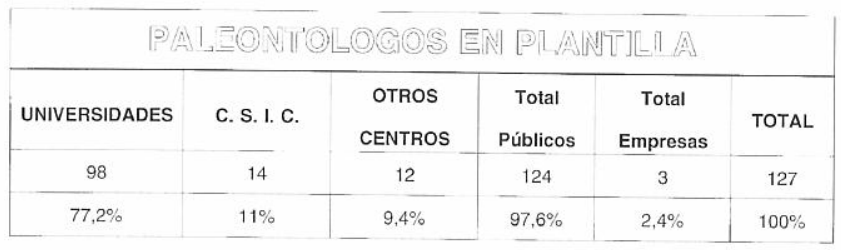

Tabla 3. Paleontólogos. Se registran los funcionarios o contratados laborales. Nótese la diferencia entre las plantillas de centros públicos y privados.

2,4\% del total. La participación relativa de la Universidad es muy alta, 77\%, y la del CSIC relativamente baja, $11 \%$; esta última responde a una política de no crecimiento de la Paleontología en esa institución dada su reorientación hacia centros y especialidades con marcado carácter de I+D. Los incrementos en ese organismo se realizan a través de plazas de museología, aunque sus titulares dediquen atención a la investigación.

\begin{tabular}{|c|c|c|c|}
\hline \multicolumn{3}{|c|}{ INVESTIGADORES } \\
\hline PLANTILLA & $\begin{array}{c}\text { ACTIVOS } \\
\text { Antes de } 1985\end{array}$ & $\begin{array}{c}\text { ACTIVOS } \\
\text { desde } 1985\end{array}$ & TOTAL \\
\hline 127 & 133 & $\frac{157}{54 \%}$ & 290 \\
\hline $44 \%$ & $46 \%$ & $100 \%$ \\
\hline
\end{tabular}

Tabla 4. Paleontólogos. Se considera investigador al detectado en las publicaciones revisadas. Se incluyen los funcionarios de otros organismos. Los datos anteriores a 1985 son de Morales \& Rivas (1987).

El número de paleontólogos aumenta significativamente, 290, si contabilizamos los que voy a denominar investigadores activos, esto es, los que se encuentran en el vaciado realizado desde 1985 . Hay que destacar la relativa juventud del colectivo pues casi la mitad han realizado su primera publicación en los últimos nueve años.

El número de investigadores activos por centros modifica algo la distribución anterior y el peso de la iniciativa privada pasa a ser insignificante (Tabla 5). Aparecen, en cambio, casi un $8 \%$ de investigadores ligados a centros de carácter no paleontológico. El crecimiento relativo del núme- ro de investigadores desde los años cuarenta sólo se puede hacer considerando los de plantilla ya que no se poseen datos sobre los activos (Fig. 2, en blanco datos de 1986; sombreado: datos actuales). A pesar de todas las previsiones pesimistas la plantilla ha crecido significativamente a partir del desarrollo universitario; sin embargo hay que volver a destacar el crecimiento basado en el desarrollo de los centros de carácter cultural o investigador patrocinados por Ayuntamientos, Diputaciones, etc

\begin{tabular}{|c|c|c|c|c|c|}
\hline \multicolumn{6}{|c|}{ DISTRIBUCION DE INVESTIGADORES } \\
\hline UNIVERSIDADES & CSIC & $\begin{array}{c}\text { OTROS CENTROS } \\
\text { DE } \\
\text { INVESTIGACION }\end{array}$ & EMPRESAS & $\begin{array}{c}\text { OTROS } \\
\text { INVESTIG. } \\
\text { Uni+Publ+Priv }\end{array}$ & TOTAL \\
\hline 203 & 31 & 31 & 3 & 22 & 290 \\
\hline $70 \%$ & $10,7 \%$ & $10,7 \%$ & $1 \%$ & $7,6 \%$ & $100 \%$ \\
\hline
\end{tabular}

Tabla 5. Paleontólogos. Distribución de los investigadores activos por centros según el tipo de estos. En "OTROS INVESTIGADORES" se contabilizan los pertenecientes a centros no universitarios y a los de la Universidad de otras Áreas de Conocimiento.

La relacion centros-investigadores se establece teniendo en cuenta los investigadores activos y los de plantilla (Figs. 1 y 2). El número de centros ha aumentado respecto a 1986, en especial los de menor tamaño (1 ó 2 investigadores). La distribución de la plantilla presenta una concentración de los investigadores en centros medios (3 a 10 investigadores) y grandes (más de 10 investigadores) aunque el crecimiento también ha sido notable en los de menor tamaño. Todo esto habla de dos comunidades diferentes, los científicos aislados y los que se integran en comunidades muy amplias, por lo general mayores que las de los países de nuestro entorno. Si se consideran los investigadores activos y se comparan con los de plantilla (Fig. 1; igual que en Fig. 2), el tamaño de los centros amplia el rango de variabilidad, pero se mantiene el tipo de distribución. Las causas son dobles, por un lado las históricas: se abren nuevos centros que, naturalmente, al inicio son de pequeño tamaño; por otro lado hay centros que no pueden crecer pues los criterios para hacerlo son las necesidades docentes o la demanda del sector servicios, que son inferiores a la capacidad de formación de los investigadores de plantilla, o al interés de los estudiantes para dedicarse a la Paleontología.

El panorama cambia si se considera la distribución de los investigadores activos por centros. El número mayor de éstos se concentra en los centros de tamaño medio y grande, en los que duplican a los de plantilla. En los centros de tamaño medio las expectativas de crecimiento, la antigüedad y el interés de sus miembros por la formación, atraen gran número de investigadores; asimismo existen especialidades que requieren mayor colaboración que otras, caso de la Paleontología de Vertebrados en relación con las excavaciones.

$\mathrm{El}$ desfase entre investigadores de plantilla e investigadores activos nace del gran número de jóvenes que realizan investigación por un período más o menos largo de tiempo y, en menor grado, de investigadores de otras áreas que trabajan esporádicamente en Paleontología. Las publicaciones analiza- 

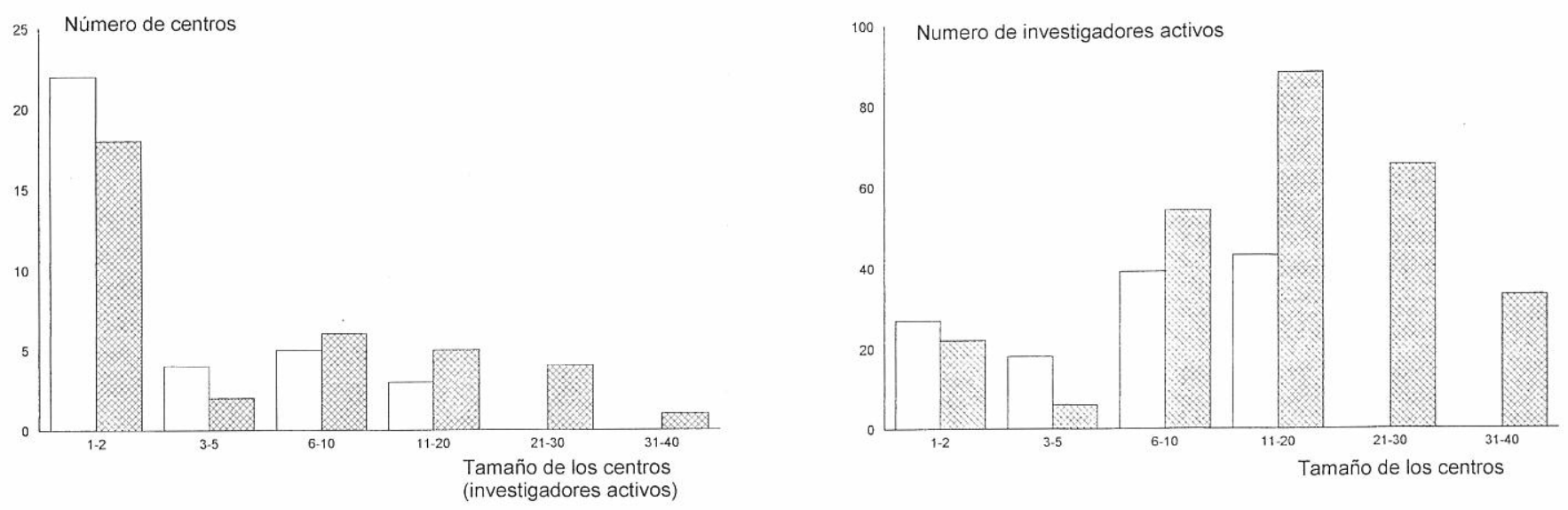

Figura 1. Distribución de investigadores activos y de centros en función del tamaño de estos contabilizando los de investigadores activos (columnas sombreadas). Las columnas en blanco representan los investigadores de plantilla.

das permiten eliminar los investigadores más eventuales (trabajos fin de carrera, etc.), y recoger a los formados aunque trabajen de forma esporádica. Hay que resaltar el gran número de becarios y doctorandos que acercan el modelo español al de otros países europeos, con una relación relativamente baja entre investigadores de plantilla y en formación o con contratos temporales. A tener en cuenta en este caso:

a.- gran diversidad en el tipo de becas. Las hay de diferentes iniciativas, públicas y privadas.

b.- flexibilidad de las relaciones de los becarios con los centros y posibilidad de encadenar diferentes tipos de becas en España y en el extranjero, pre y postdoctorales, y de todas ellas con otras figuras de investigadores o profesores en formación.

Respecto a lo primero hay que resaltar que en los últimos años han aparecido becas de: empresas, universidades, OPIs, fundaciones, proyectos públicos, entidades provinciales, entidades locales, así como de un muy importante número de entes autonómicos de diferente tipo. Sólo estas últimas han duplicado el número de becarios en algunas regiones. A lo anterior hay que añadir la mayor duración de las becas que hace que existan becarios con más de nueve años de trabajo. Las becas y las estancias en el extranjero han mejorado de forma significativa la formación de los investigadores y, con ello, los índices de publicación y el acceso más rápido a las revistas de más calidad.

Los indicadores socio-económicos llevan a suponer que no se debe esperar un incremento de los investigadores en el futuro y que, como ha ocurrido en otros países, algunas plantillas se reduzcan. La situación presupuestaria tiende a disminuir el mecenazgo de entes regionales y locales. Esta impresión emperora cuando se analiza la trayectoria de la Paleontología en el C.S.I.C. Alrededor de ese organismo hay más de 30 investigadores y sólo la cuarta parte son de plantilla, que por otro lado no tiene previsiones de crecimiento específico. En la Universidad, a pesar de la disminución en la tasa de crecimiento, se ha podido absorber en plantilla o en plazas contratadas un gran número de becarios y profesores en formación. Han aumentado los investigadores que obtienen financiaciación a partir de proyectos y contratos de investigación, o de un trabajo a tiempo parcial, tanto dentro como fuera de la Universidad y que dedican una parte importante de
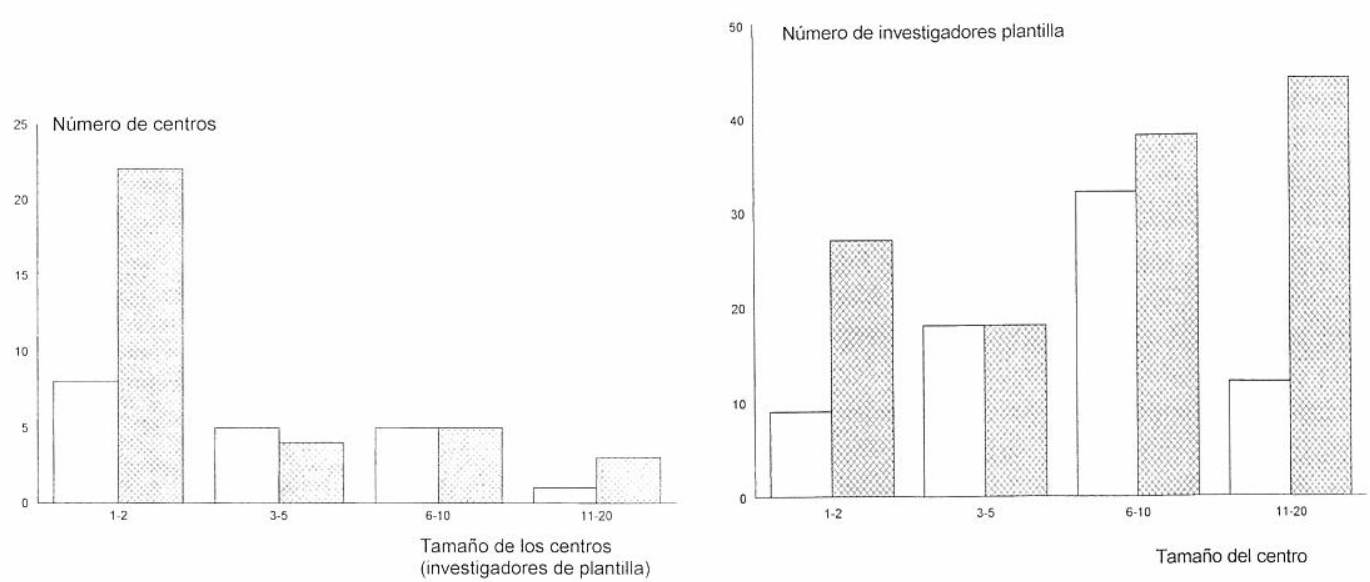

Figura 2. Distribución de los investigadores de plantilla y centros en función del tamaño de estos teniendo en cuenta sólo los investigadores de plantilla (columnas sombreadas). En columnas blancas los datos de 1985. 


\begin{tabular}{|c|c|c|c|c|c|c|c|c|c|c|}
\hline \multicolumn{2}{|c|}{ TEMAS } & $\mathbf{Q}$ & $\mathrm{N}$ & $\mathbf{P}$ & $\mathrm{K}$ & $\mathrm{J}$ & $\mathbf{T}$ & PS & $\mathrm{PI}$ & PRC \\
\hline \multicolumn{2}{|r|}{ PALEOBOTANICA } & $\mathrm{xx} \times \bullet$ & $X X X_{\theta}$ & $x x$ & $\mathrm{x}$ & No & No & $x x_{\theta}$ & & \\
\hline \multicolumn{2}{|c|}{ PALEOICNOLOGIA } & No & $x \times x=$ & & $\mathrm{N}$ & NNE & & $N$ & $\mathrm{~N}$ & $\mathrm{~N}$ \\
\hline \multirow{5}{*}{$\begin{array}{l}V \\
V \\
\text { E } \\
T \\
E \\
B \\
B \\
A\end{array}$} & Humana & $x x_{0}$ & & & & & & & & \\
\hline & Mamiferos & $x x x$ & $x X x_{\theta}$ & $x x_{\bullet}$ & & & & & & \\
\hline & Aves & $\mathrm{N}$ & $\mathrm{N}$ & & Ne & & & & & \\
\hline & Rept.+ Anf. & $\mathrm{N}$ & $x$ & $x x_{0}$ & $x x_{0}$ & & $\mathrm{~N}$ & & & \\
\hline & Peces & $\mathrm{N}$ & $\mathrm{N}$ & & & & & $\mathrm{N}$ & & \\
\hline \multirow{9}{*}{\begin{tabular}{l||l||} 
& \\
$N$ & || \\
$V$ \\
$E$ \\
$R$ \\
$T$ \\
$E$ \\
$E$ \\
$B$ \\
$R$ \\
$A$ \\
$D$ \\
$D$ \\
$O$ \\
$S$
\end{tabular}} & Graptolites & & & & & & & & $x_{\bullet}$ & \\
\hline & Equinodermos & & & & & & & $x$ & $\mathrm{~N}$ & \\
\hline & Trilobites & & & & & & & $x$ & $x \times x=$ & \\
\hline & Moluscos & $x_{0}$ & $x \times x_{\theta}$ & $\mathrm{x}$ & $x x$ & $x$ & $x x_{0}$ & 0 & $x_{\theta}$ & \\
\hline & Cefalópodos & & & & $x x_{\bullet}$ & $x x_{0}$ & $x$ & $x x$ & & \\
\hline & Braquiopodos & & & & $x$ & $x x_{0}$ & & $x_{\bullet}$ & $x_{\theta}$ & \\
\hline & Briozoos & $N$ & $x$ & 0 & & & & $\mathrm{~N}$ & No & \\
\hline & Cnidr.+ Arq. & & NNN@ & $\mathrm{N}$ & & $\mathrm{N}$ & $\mathrm{N}$ & $x x_{0} \theta$ & $x X_{0}$ & \\
\hline & Artr.Insect. & $\mathrm{N}$ & NNE & No & & $x$ & & & & \\
\hline \multirow{6}{*}{\begin{tabular}{l||}
$M$ \\
$I$ \\
$C$ \\
$\mathrm{~A}$ \\
$\mathrm{O}$ \\
$\mathrm{P}$ \\
$\mathrm{A}$ \\
$\mathrm{L}$ \\
$\mathrm{E}$ \\
$\mathrm{O}$ \\
$\mathrm{N}$
\end{tabular}} & Palin+Acrit & $x$ & $x$ & 0 & $x$ & $x$ & & $x$ & $x x$ & \\
\hline & Algas & Ne & No & $x$ & $x \times 0$ & 0 & $x$ & $x$ & $x$ & \\
\hline & Conodontos & & & & & & $x$ & $x x$ & $\mathrm{~N}$ & \\
\hline & Ostrácodos & & NN & & NN & $x$ & & $x$ & $\mathrm{~N}$ & \\
\hline & Nannoplanct. & $x$ & $x x$ & NNO & $x \times x_{0}$ & & & $\mathrm{~N}$ & NNN & \\
\hline & Foraminif. & $x \times x_{0}$ & $x \times x_{\odot}$ & $x \times x_{0}$ & $x \times X_{0}$ & $\mathrm{O}$ & No & NN & & \\
\hline \multicolumn{2}{|c|}{$\begin{array}{l}\text { PALEOBIOLOGIA } \\
\text { TAFONOMIA } \\
\end{array}$} & 41 & & & & & & & & \\
\hline \multicolumn{2}{|c|}{$\begin{array}{l}\text { HIST, PEDAGOGIA } \\
\text { PATRIMONIO }\end{array}$} & 22 & & & & & & & & \\
\hline
\end{tabular}

Tabla 6. Temática. Se han sombreado los temas abordados en los últimos años para resaltar la evolución de la Paleontología española. Un símbolo ( $\mathrm{X}$ o $\mathrm{N}$ ) indica un investigador; dos símbolos: dos o tres; tres símbolos: más de tres, normalmente más de cinco.

su tiempo a la investigación. Este colectivo no parece que pueda desaparecer; es más, las condiciones sociales favorecen su crecimiento. La masificación universitaria, que será aun mayor en los próximos años, va a incrementar las plantillas de profesorado, y en el mismo sentido apuntan las nuevas formas de docencia (cursos cortos, horarios y fechas diferentes a las actuales,etc.). Habrá que reciclar todo tipo de profesionales, incluidos los docentes, de forma mucho más intensa y frecuente, lo que aumentará la demanda de profesores.

No debemos dejar sitio al pesimismo; el aumento del sector servicios, en relación con la mejora del bienestar social, debe suponer un incremento de la oferta cultural y la Paleontología es parte de esa oferta. Que se materialice o no depende de nosotros mismos y de nuestra organización. Por otro lado, la mayor capacidad de formación atraerá a un mayor número de investigadores extranjeros en formación, que en algunos centros ya superan a los nacionales. La solidaridad con el tercer mundo supone la enseñanza a sus miembros en nuestro país y las demandas y movimientos sociales van camino de incrementar significativamente los fondos dedicados a la cooperación para el desarrollo.

\section{PRODUCCION}

La temática de la producción científica se ha resumido en el cuadro adjunto (Tabla 6), en el que también se indican, de forma muy general, la mayor o menor atención que se dedica a cada grupo de organismos. Se cubre el estudio de la mayor parte de los grupos fósiles y se hace bajo diferentes aspectos: bioestratigráfico, taxonómico, evolutivo, paleoecológico, tafonómico, etc. A esto hay que añadir un esfuerzo considerable para tratar temas generales, no ligados a un grupo de organismos concreto, que se ha multiplicado por ocho en los últimos años. El mismo esfuerzo se deduce del gran número de temas iniciados en los últimos años que habla en favor de la juventud científica de nuestra comunidad. Hay un intento serio de cubrir necesidades bioestratigráficas básicas a partir de escalas múltiples, y también una migración inteligente (pues hay más recursos disponibles) hacia el estudio de grupos del Neógeno y Cuaternario, y de los que tienen interés paleoecológico frente a los que lo tienen bioestratigráfico (Tabla 7).

Hay un avance importante de la Micropaleontología (Tabla 8) que absorbe casi el 30\% del total de las firmas de

\begin{tabular}{|c|c|c|}
\hline TEMAS & \multicolumn{2}{|c|}{ AUTORES } \\
\hline $\begin{array}{c}\text { Micropaleontología } \\
\text { incluye } \\
\text { Palinología }\end{array}$ & 132 & $28,1 \%$ \\
\hline Paleoicnología & 21 & $4.5 \%$ \\
\hline $\begin{array}{c}\text { Paleontología } \\
\text { de Vertebrados } \\
\text { incluye P. humana }\end{array}$ & 101 & $21,5 \%$ \\
\hline $\begin{array}{c}\text { Paleontología } \\
\text { de Invertebrados } \\
\text { incl. bioconstrucciones }\end{array}$ & $(100+11)$ & $24,5 \%$ \\
\hline $\begin{array}{c}\text { Paleobotánica } \\
\text { incluye Palinología }\end{array}$ & 38 & $8 \%$ \\
\hline $\begin{array}{c}\text { Tafonomomía } \\
\text { Paleobiología }\end{array}$ & 41 & $8,7 \%$ \\
\hline $\begin{array}{c}\text { Pedagogía } \\
\text { Historia } \\
\text { Patrimonio }\end{array}$ & 22 & $4,7 \%$ \\
\hline
\end{tabular}

\section{La relación autor-tema/autor es 1,6}

Tabla 7. Temática. "Autor" es el firmante de algún trabajo sobre el tema. Los datos indican que cada investigador publica en algo más de 1,6 temas. La Palinología se incluye en dos temas pues es difícil de separar de ambos. 


\begin{tabular}{|c|c|c|c|c|c|}
\hline \multicolumn{7}{|c|}{ MICROP ALEONTOLOGIA } \\
\hline temas/trab \% & PALAEONTOLOGY & $\begin{array}{c}\text { REV. ESP. } \\
\text { PALEONTOLOGIA }\end{array}$ & MICROPALEONTOLOGY & $\begin{array}{c}\text { REV. ESP } \\
\text { MICROPAL. }\end{array}$ & $\begin{array}{c}\text { Este } \\
\text { Trabajo }\end{array}$ \\
\hline Foraminíferos & 7 & 87,5 & 38 & 52 & 37,7 \\
\hline Nannoplancton & 12 & --- & 13 & 7 & 16,6 \\
\hline Palinología & 18,5 & --- & 7 & 17,4 & 20 \\
\hline Otros grupos & --- & 6,2 & 18 & 2,6 & 1,5 \\
\hline Conodiagelados & 15 & -- & 1 & 1 & 5,3 \\
\hline Ostrácodos & 15 & -- & 9 & 8 & 12,1 \\
\hline Algas & 25,5 & 6,2 & 14 & 2 & 7,5 \\
\hline
\end{tabular}

Tabla 8. Análisis de las publicaciones de temática micropaleontológica. En el apartado "Este Trabajo" se indican las medias respecto a todas las revistas consultadas en este trabajo, teniendo en cuenta sólo los trabajos de temática micropaleontológica.

trabajos (en el estudio de la producción se contabilizan las firmas de los investigadores, no los trabajos publicados); le siguen la Paleontología de Invertebrados y la de Vertebrados con algo más del $20 \%$ cada una. El incremento de la Paleontología de Vertebrados ha sido espectacular, y es mayor que la que muestran las cifras, pues en este caso los investigadores se dedican casi exclusivamente al grupo, frente a lo que ocurre con los Invertebrados o la Micropaleontología; los investigadores que se dedican a éstos últimos publican sobre más de un tema. Hay que destacar también el crecimiento de la Paleobotánica y sobre todo el de la Paleontología básica que indica la madurez de la comunidad.

Cabe pensar que en el futuro la Micropaleontología se mantenga, e incluso se desarrolle más para cubrir nuevos grupos; los micropaleontólogos constituyen una comunidad activa que avanza en los estudios tafonómicos y paleoecológicos, que les permiten completar los que hasta ahora eran sus intereses principales: bioestratigrafía, taxonomía, morfología, extinciones. El análisis de las publicaciones españolas y de los españoles en Micropaleontología permite detectar algunas diferencias respecto a otras comunidades científicas (Tabla 8). La dedicación a los foraminíferos es mayor que en el resto de los países, y hay un desfase en algas; otros grupos como radiolarios, etc., se tratan muy poco en España. También existe un desequilibrio en la temática del nanoplancton, muy sesgada hacia el calcáreo.

Peor suerte hay que esperar para la Paleontología de Vertebrados pues va a resultar difícil, por lo caro del proceso, mantener los actuales niveles de investigadores noveles. En sentido positivo hay que señalar el carácter vocacional de esta rama y su dependencia y financiación de los departamentos de cultura y patrimonio de las autonomías. El crecimiento de las publicaciones en los últimos años ha sido espectacular y hay un peso muy grande de los trabajos sobre
Mamíferos neógenos. Esto es más acusado cuando se analizan el total de publicaciones (Gregory et al., eds. 1983; 1988; 1989a; 1989b; 1991; 1992 y 1993) (Tablas 9 y 10), y no sólo las consultadas en este trabajo. La comparación entre los niveles de publicación del año 1980 y la actualidad evidencia un mayor peso de la Paleontología de Vertebrados española en el contexto internacional, en especial en el estudio de los mamíferos neógenos.

\begin{tabular}{|c|c|c|c|}
\hline \multicolumn{4}{|c|}{$\begin{array}{c}\text { PALEONTOLOGIA DE VERTEBRADOS } \\
\text { temas en publicaciones }(\%)\end{array}$} \\
\hline & FRANCIA & ITALIA & ESPAÑA \\
\hline P. Humana & $8,3 \%$ & $13,7 \%$ & $11,4 \%$ \\
\hline Mamíferos & $56,8 \%$ & $56,5 \%$ & $66 \%$ \\
\hline Aves & $5 \%$ & $1,3 \%$ & $4 \%$ \\
\hline Reptiles y Anfibios & $18 \%$ & $10,2 \%$ & $13,2 \%$ \\
\hline Peces & $11,9 \%$ & $18,2 \%$ & $5,4 \%$ \\
\hline
\end{tabular}

Tabla 9. Análisis de las publicaciones de Paleontología de Vertebrados de los nueve últimos años.

Del cuadro inicial también parece desprenderse un cambio en la tendencia de los trabajos de Paleontología de Invertebrados, con mayor atención a grupos de interés paleoecológico. 


\section{PALEONTOLOGIA DE VERTEBRADOS}

\begin{tabular}{|c|c|c|}
\hline & 1980 & $1985-90$ \\
\hline FRANCIA & $41 \%$ & $45 \%$ \\
\hline ITALIA & $37,5 \%$ & $22,2 \%$ \\
\hline ESPAÑA & $21,5 \%$ & $32,8 \%$ \\
\hline
\end{tabular}

La producción científica total se ha incrementado en un $15 \%$

Tabla 10. Evolución de la temática en Paleontología de Vertebrados en los tres países. La producción científica total de los tres ha aumentado un $15 \%$ y el peso de España en la producción se ha incrementado en un $52 \%$.

La dedicación y producción de nuestros investigadores es intensa, pues publican de forma continuada (investigadores permanentes) ciento sesenta y nueve respecto a una plantilla de sólo ciento veintisiete investigadores. El índice de publicación o firma para el total de los investigadores es alto dado el escaso número de revistas españolas analizadas (2,7 por investigador) (Tablas 11 y 12). Este índice aumenta cuando se tienen en cuenta todas las publicaciones, y más cuando se eliminan los "investigadores ocasionales", esto es a los que sólo se le ha contabilizado un trabajo desde 1985. Para el resto, el índice de publicación es 4,4, el 13,1 \% en revistas paleontológicas incluidas en el SCI . Naturalmente a lo anterior hay que aplicarle una corrección muy importante, si se quiere tener en cuenta la producción científica total, de dos a cuatro veces superior. Este cálculo se basa en los datos obtenidos para un grupo de investigadores (Univ. de Granada) en este trabajo, a partir de las revistas vaciadas, y los datos que suministra Linares (1993) sobre el total de las publicaciones del mismo grupo.

\begin{tabular}{|c|c|c|c|c|}
\hline \multicolumn{5}{c}{ DEDICACION } \\
\hline $\begin{array}{c}\text { TOTAL DE } \\
\text { INVESTIGADORES }\end{array}$ & $\begin{array}{c}\text { Investigadores } \\
\text { ocasionales }\end{array}$ & \multicolumn{2}{c|}{$\begin{array}{c}\text { Investigadores } \\
\text { no ocasionales }\end{array}$} \\
\hline 290 & \multicolumn{2}{|c|}{121} & \multicolumn{2}{|c|}{169} \\
\hline & $\begin{array}{c}\text { antes } \\
1985\end{array}$ & desde & antes & desde \\
\hline & 24 & 97 & 1885 & 1985 \\
\hline & & & 109 & 60 \\
\hline
\end{tabular}

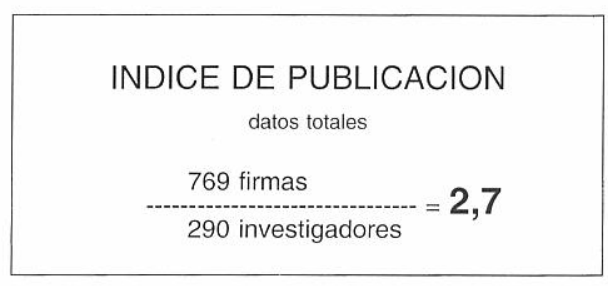

Tabla 11. Investigadores y producción. Los investigadores ocasionales son los que sólo han publicado un trabajo. Nótese el gran incremento de los ocasionales desde 1985.

\begin{tabular}{|c|c|c|c|c|c|c|c|}
\hline \multicolumn{8}{|c|}{ PRODUCCION CIENTIFICA } \\
\hline & & FIRMAS & INVEST. & $F / l$ & $\begin{array}{c}\text { FIRMAS } \\
\text { SCI }\end{array}$ & $\begin{array}{c}\text { FIRMAS } \\
\text { SCI }\end{array}$ & $\begin{array}{l}\mathrm{F} / \mathrm{l} \\
\mathrm{SCl}\end{array}$ \\
\hline $\begin{array}{l}\text { CONJUNTO } \\
35 \text { CENTROS }\end{array}$ & \begin{tabular}{|l|} 
Totales \\
Ocasionales \\
No Ocasionales
\end{tabular} & $\begin{array}{l}866 \\
121 \\
745\end{array}$ & $\begin{array}{l}290 \\
121 \\
169\end{array}$ & $\begin{array}{c}3 \\
1 \\
4,4\end{array}$ & $\begin{array}{c}97 \\
- \\
97\end{array}$ & $\begin{array}{c}11,2 \\
-\overline{13,1}\end{array}$ & $\begin{array}{c}0,33 \\
-\cdot \\
0,57\end{array}$ \\
\hline $\begin{array}{c}4 \\
\text { CENTROS } \\
\text { MAYORES }\end{array}$ & \begin{tabular}{|l|} 
Totales \\
Ocasionales \\
No Ocasionales
\end{tabular} & $\begin{array}{c}280 \\
36 \\
244\end{array}$ & $\begin{array}{l}97 \\
36 \\
61\end{array}$ & $\begin{array}{c}2,9 \\
1 \\
4 \\
\end{array}$ & $\begin{array}{l}55 \\
- \\
55\end{array}$ & $\begin{array}{c}19,6 \\
-- \\
22,5\end{array}$ & $\begin{array}{c}0,56 \\
-\overline{-} \\
0,90\end{array}$ \\
\hline $\begin{array}{l}31 \text { CENTROS } \\
\text { RESTANTES }\end{array}$ & \begin{tabular}{|l|} 
Totales \\
Ocasionales \\
No Ocasionales
\end{tabular} & $\begin{array}{c}586 \\
85 \\
471\end{array}$ & $\begin{array}{c}193 \\
85 \\
108\end{array}$ & $\begin{array}{l}3 \\
1 \\
4,4\end{array}$ & $\begin{array}{l}42 \\
-- \\
42\end{array}$ & $\begin{array}{l}7,1 \\
- \\
8,9\end{array}$ & $\begin{array}{c}0,22 \\
-- \\
0,39\end{array}$ \\
\hline
\end{tabular}

Tabla 12. Producción científica por autores (no trabajos) y proporción de trabajos firmados por cada investigador. Nótese la mayor incidencia de los tres centros de mayor tamaño.

Aunque no sea un índice de calidad definitivo, la incidencia en revistas de gran prestigio es significativa. En las analizadas se llega a 0,57 firmas por investigador. Al no poder hacer una estimación de la incidencia de este tipo de publicación respecto a la edad o a la antigüedad de los investigadores se ha hecho respecto al tipo de centros, y se obtienen unos resultado realmente interesantes, (Tabla 12). En los de mayor tamaño el índice de publicación en revistas españolas es similar al de los centros pequeños y medianos, mientras que el referido a revistas extranjeras es casi tres veces mayor. En la tabla 6, se señalan los temas en los que hay una mayor incidencia internacional. El 80\% de los temas reseñados con mayor número de investigadores son conocidos en las publicaciones del SCI y lo mismo ocurre con el $15 \%$ de los que son abordados por un solo investigador. Por otra parte, el $37 \%$ de los temas cuyo estudio se ha iniciado en los últimos años están contenidos en parte en revistas internacionales. Esta incidencia tan temprana se explica por la mejor formación de los nuevos investigadores, por una selección en clave de calidad de los grupos receptores de becarios, de la dedicación exclusiva de estos a la investigación, de las facilidades para completar su formación en otros centros, etc. También hay que tener en cuenta la reconversión de investigadores ya formados que trabajan en una especialidad nueva.

Queda por analizar el carácter de las publicaciones españolas. En el cuadro aparecen los datos de los autores españoles y extranjeros que publican en revistas españolas; que los segundos constituyan un $30 \%$ parece una cifra importante (Tabla 13), de los que casi el $8 \%$ corresponde a la Revista Española de Micropalentología, de gran arraigo entre los especialistas extranjeros.

\begin{tabular}{|c|c|c|c|c|}
\hline \multicolumn{5}{|c|}{ REVISTAS ESPAÑOLAS } \\
\hline & \multicolumn{2}{|c|}{ Todas las revistas } & \multicolumn{2}{|c|}{ Sin R.E.Micropaleo. } \\
\hline $\begin{array}{c}\text { Total firmas } \\
\text { en Revistas Españolas }\end{array}$ & 1109 & & 957 & \\
\hline $\begin{array}{c}\text { Colaboraciones de } \\
\text { investigadores españoles }\end{array}$ & 769 & $69,3 \%$ & 740 & $77,3 \%$ \\
\hline $\begin{array}{c}\text { Colaboraciones de } \\
\text { investigadores extranjeros }\end{array}$ & 340 & $30,7 \%$ & 217 & $22,7 \%$ \\
\hline
\end{tabular}

Tabla 13. Autores extranjeros en publicaciones españolas. Se hace una excepción con la R.E. de Micropaleontología por el gran número de extranjeros que colabora en la misma. 


\section{DEMANDAS}

Las demandas se conforman por los intereses de las diferentes comunidades relacionadas con la Paleontología: paleontológicas internacional y nacional, de otras ciencias próximas, de los centros de Paleontología, de los sectores socio-culturales, de los económicos, etc.

\section{COMUNIDAD PALEONTOLÓGICA}

\section{Comunidad Paleontológica internacional (Tabla 14)}

Hoffman (1990) puede ser un buen intérprete de los intereses de los paleontólogos. En su análisis proyectivo separa la Paleontografía de la Paleontología Teórica. La moderna Paleontografía incluye estudios que se consideraban teóricos hace unos años, lo que da a esta rama un gran contenido básico y general. La evolución de la Paleontología, las técnicas avanzadas de análisis y los aportes de otras ciencias próximas permiten abordar de forma prioritaria:

- estudios sobre organismos enigmáticos: tabulados, estromatopóridos, conodontos, faunas peculiares, etc.

- formas y modos de vida de los organismos extintos a través de modelos paradigmáticos.

- paleocomunidades, medios de vida y su evolución.

- eventos sucesivos y ciclos ecológicos.

- reconstrucción de filogenias por análisis morfológicos, taxonómicos, ecológicos, biogeográficos y bioestratigráficos.

- estructura biogeográfica y ecológica de la biosfera y su evolución.

- etc.

\begin{tabular}{|c|c|c|c|}
\hline \multicolumn{4}{|c|}{$\begin{array}{c}\text { DEMANDAS } \\
\text { COMUNIDAD PALEONTOLOGICA INTERNACIONAL }\end{array}$} \\
\hline T. GENERALES & T. CONCRETOS & METODOS & TECNICAS \\
\hline $\begin{array}{l}\text { ESQUEMAS EVOLUTIVOS } \\
\text { PROCESOS EVOLUTIVOS } \\
\text { LEYES EVOLUTIVAS } \\
\text { Equilibrio interrumpido } \\
\text { Selección de especies } \\
\text { Diversificación taxonómica } \\
\text { Extinciones en masa } \\
\text { etc. } \\
\text { HISTORIA DE LA VIDA }\end{array}$ & $\begin{array}{l}\text { Organismos enigmáticos } \\
\text { Morfología } \\
\text { Selección } \\
\text { Formas de vida } \\
\text { Estructura de la biosfera } \\
\text { Paleobioquimica } \\
\text { Pal. celular } \\
\begin{array}{l}\text { Tafonomía } \\
\text { tafofacies } \\
\text { f. lagerstätten } \\
\text { diagénesis }\end{array}\end{array}$ & 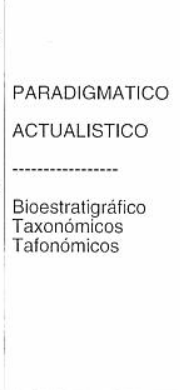 & $\begin{array}{l}\text { Geoquimicas } \\
\text { Q. Isotópica } \\
\text { M. electrónica } \\
\text { A. numéricos } \\
\text { A. estadísticos } \\
\text { Reproducciones } \\
\text { a escala } \\
\text { etc., etc... }\end{array}$ \\
\hline
\end{tabular}

Tabla 14. Intereses más destacados de los paleontólogos. Se han obtenido de trabajos especializados y de lo tratado en manuales y en las publicaciones de mayor difusión (SCI).

Esto supone un gran desarrollo de los estudios morfológicos desde los más variados puntos de vista: teórico, construccional, funcional... A partir de ellos se entra en el análisis de la selección y la limitación de las morfologías en el curso de la evolución (Alberch, 1991), así como de modelos evolutivos: evolución paralela, convergente, etc. Parece evidente que cuando falta la información bioquímica, genética, fisiológica, anatómica de las partes blandas, etc., hay que desarrollar en extenso el análisis de la información disponible (morfología esquelética). En la profundización de esta rama y metodología hay un futuro prometedor y específico de la Paleontología. Lo anterior se complementa con estudios sobre Paleontología Molecular y Celular, puntuales, por lo difícil de la conservación de los fósiles y aplicación de la metodología, pero que suministran a la Paleontología verificaciones y apoyos que permiten calibrar los obtenidos a partir de la morfología (Runnegar \& Schopf, con., 1988).

El desarrollo de la Tafonomía es un tema de índole muy diferente. Su estudio, una vez establecidas las bases teóricas (Fernández López, 1991; ed., 1993) se centra en la Tafonomía aplicada (Allison \& Briggs, eds., 1991; Kidwell, 1991):

a.- Tafofacies y asociaciones fósiles para establecer su correlación con las biofacies y las comunidades. Hay una retroalimentación tafonómica o cambio ambiental inducido por el tipo de acúmulo de restos de organismos.

b.- Procesos fosildiagenéticos que permiten establecer la fiabilidad morfológica, mineralógica etc. de los fósiles, a la vez que se convierten en marcadores geológicos importantes.

c.- Depósitos de enterramiento rápido y de medios estancados, ámbar, etc. que dan lugar a conservaciones excepcionales. Estas permiten interpretaciones de formas de cuerpo blando o conocimiento de partes blandas de otros organismos, así como información sobre la composición de comunidades y la forma de vida de algunos organismos y la relación entre las bioturbaciones y los organismos que las producen (Brett \& Speyer, 1990 ; Seilacher, 1990).

Asimismo la Tafonomía es un instrumento fundamental en el análisis de cuencas sedimentarias. La pérdida de información biológica se ve compensada por el aumento de la tafonómica, de gran interés en las reconstrucciones geológicas.

Dentro del apartado paleontográfico hay que destacar los aspectos metodológicos:

- la aplicación cada vez más generalizada de nuevas técnicas de análisis.

- los esfuerzos para obtener una escala bioestratigráfica fiable y una taxonomía adecuada para el estudio de los modelos ecológicos, morfológicos, ontogenéticos, evolutivos, etc.

La Paleontología teórica se interesa en cuestiones básicas:

- ¿Por qué son así los modelos de árboles filogenéticos?

- ¿Cómo operan los procesos evolutivos?

- ¿Cuáles son las leyes universales de la evolución?

Estas cuestiones se corresponden con las grandes preguntas que Gould (1977) considera "eternas". Más interesante, por próximo, puede resultar el análisis y la verificación de las teorías e hipótesis más modernas, propias de la Paleontología, gracias a las cuales despegó nuestra ciencia en los años setenta (Sepkoski, 1978; Briggs \& Crowther, eds., op. cit; Lamolda et al, eds, 1988; Raup, 1990; Kauffman \& Walliser, 1990; Jablonski, 1986, Hoffman, 1988, etc.): 
- Equilibrio interrumpido a intervalos.

- Selección de especies.

- Diversificación taxonómica.

- Extinciones en masa.

Parece que en el futuro de la Paleontología, al menos a corto y medio plazo, no se van a producir grandes teorías, ni que, excepto en el tema de las extinciones en el que los esfuerzos son muy amplios, se puedan solucionar las controversias planteadas. Eldredge (1989) ha hecho algunas propuestas que necesitan de apoyo empírico. Las unidades ecológicas pueden tener mejor suerte que las genealógicas en el registro fósil ya que los cambios ambientales tienen mejor registro geológico que los orgánicos

Una verificación de lo que significan estas propuestas teóricas, producto de la elucubración, se obtiene al analizar lo publicado en los últimos años. La Paleontografía y la Paleontología Teórica siguen canales de difusión muy diferentes. Los aspectos teóricos generales interesan a toda la comunidad científica, mientras que los paleontográficos prioritariamente a la paleontológica. En las grandes revistas de la especialidad los temas más frecuentes son:

- Faunas, i.e. Burgess Shale, Ediacara, conodontos, organismos de cuerpo blando, etc.

- Morfología; Morfología Funcional; crecimiento.

- Morfología-Taxonomía-Evolución: diversidad morfológica y taxonómica, tasas evolutivas, simulación evolutiva, etc.

- Paleoecología. Predación; ecosistemas marinos; ecosistemas marinos profundos. Paleoecología general; modos de vida de organismos.

- Tafonomía tanto de unidades como de organismos.

En la mayor parte de los casos se utiliza la metodología paradigmática en vez de la actualística. Han aumentado los estudios sobre grupos fósiles "minoritarios", y los trabajos sobre los grupos clásicos se refieren a los que no tienen representantes actuales; en ammonites se pone en duda la validez de las analogías en relación a Nautilus y se prefiere trabajar con modelos.

Las aportaciones sobre Paleontología Teórica se producen en circuitos diferentes, fundamentalmente publicaciones de grandes sociedades científicas, revistas generales de gran prestigio (Science, Nature) y sobre todo en monografías y libros colectivos.

El acceso futuro de los paleontólogos españoles a esos cauces puede ser lento, largo y difícil. Por el contrario, los aportes a la Paleontografía pueden continuar siendo importantes. Los estudios sobre aves, homínidos, insectos, etc., pertenecen a esta línea, y el creciente interés por los yacimientos de acúmulo rápido apunta en el mismo sentido. Se está alcanzando un nivel más que aceptable en Tafonomía práctica, en trabajos interpretativos, donde lo ecológico, lo tafonómico y lo bioestratigráfico se integran en reconstrucciones amplias. Lo mismo se puede decir de los estudios sobre Morfogénesis que cabe esperar cobren auge sobre las bases actuales.

La comunidad científica internacional nos demanda una producción de calidad, y de los datos se desprende un aumento en la misma. Gustaría pensar que es más producto de nuestra evolución intrínseca que de una política científica; asumamos, por humildad, un equilibrio entre ambas causas.

\section{Comunidad nacional, regional, etc. (Tabla 15)}

Los grupos de organismos fósiles en que trabajan los paleontólogos españoles (Tabla 6), suponen la superación de las deficiencias detectadas en 1987 (Morales \& Rivas, op. cit). Se han cubierto suficientemente: nanoplancton, corales mesozoicos y terciarios, Paleoicnología, Palinología, ostrácodos, conodontos, micromamíferos; se ha avanzado en vertebrados no mamíferos, grupos minoritarios (i.e. insectos), algas, foraminíferos bentónicos, moluscos, etc.

Los resultados son menos optimistas respecto a los temas generales que la comunidad científica española ha considerado interesantes (Morales \& Rivas, op. cit.; Aguirre, coord., op. cit.; Ramírez del Pozo, op. cit.; Truyols \& López Martínez, op. cit.), o las que se proponen en este trabajo. Algunos son abordables en mejores condiciones que otros y dependen de: la existencia de un registro fósil, la preparación de los investigadores y de los intereses y ocupación de éstos.

El primer considerando constriñe el estudio de los grupos minoritarios (formas de cuerpo blando, etc.); el segundo obliga a un ritmo lento por el largo tiempo de formación de un especialista. La actitud de los investigadores permitirá completar las carencias (enfoques paleobiológicos de los trabajos, trabajos tafonómicos, etc.) y desarrollar equipos multidisciplinares al tiempo que contar con investigadores que

\begin{tabular}{|c|c|c|c|c|}
\hline \multicolumn{5}{|c|}{ TEMAS PROPUESTOS EN ESPAÑA } \\
\hline 1987 & 1989-1992 & Este trabajo & $\begin{array}{l}\text { Aspectos } \\
\text { generales }\end{array}$ & $\begin{array}{c}\text { Grupos } \\
\text { concretos }\end{array}$ \\
\hline $\begin{array}{l}\text { REGISTRO FOSIL } \\
\text { (ver cuadro) }\end{array}$ & $\begin{array}{l}\text { Paleobotánica } \\
\text { Micropaleontología } \\
\text { Mamíferos } \\
\text { Paleontología Humana }\end{array}$ & $\begin{array}{l}\text { Diatomeas, etc. } \\
\text { Inverteb. minoritarios } \\
\text { Otros Vertebrados }\end{array}$ & & $\begin{array}{l}\text { xxxxo } \\
\text { ooooo } \\
\text { xxxxo } \\
\text { xoooo } \\
\text { xxxxx } \\
\text { xxxxx } \\
\text { xxooo } \\
\end{array}$ \\
\hline TAFONOMIA & TAFONOMIA (teor.) & $\begin{array}{l}\text { Reconstrucciones } \\
\text { Bioestratigrafia }\end{array}$ & $x x x x x$ & $\begin{array}{l}\text { xoooo } \\
\text { xooooo }\end{array}$ \\
\hline $\begin{array}{l}\text { MORFOLOGIA Y } \\
\text { ONTOGENIA }\end{array}$ & $\begin{array}{l}\text { Morfogénesis } \\
\text { Microtexturas } \\
\text { Histología }\end{array}$ & $\begin{array}{l}\text { Microtexturas } \\
\text { Histología }\end{array}$ & $\begin{array}{l}\mathrm{xx} \times 000 \\
\mathrm{x}\end{array}$ & $\begin{array}{l}\text { xooo } \\
\text { xoooo } \\
\text { ooooo }\end{array}$ \\
\hline $\begin{array}{l}\text { POBLACIONES } \\
\text { COMUNIDADES }\end{array}$ & $\begin{array}{l}\text { Relaciones sustrato } \\
\text { Paleoicnología } \\
\text { Paleoclimatología } \\
\text { Comunidades }\end{array}$ & $\begin{array}{l}\text { Relaciones sustrato } \\
\text { Paleoclimatología } \\
\text { de medios marinos }\end{array}$ & $\begin{array}{l}x x x o o \\
x x\end{array}$ & $\begin{array}{l}00000 \\
x x x x x \\
x \text { xooo }\end{array}$ \\
\hline PALEOBIOGEOGRAFIA & $\begin{array}{l}\text { P. TEORICA } \\
\text { ccológica } \\
\text { histórica } \\
\text { Dispersiones grupos }\end{array}$ & $\begin{array}{l}\text { P. ecológica } \\
\text { P. histórica } \\
\text { Dispersiones grupos }\end{array}$ & $x x$ & $\begin{array}{l}\text { oo } \\
\text { ooooo } \\
\text { xxxoo } \\
\text { xxooo }\end{array}$ \\
\hline $\begin{array}{l}\text { PALEONTOLOGIA } \\
\text { EVOLUTIVA }\end{array}$ & $\begin{array}{l}\text { Filogenias } \\
\text { Ritmos evolutivos } \\
\text { Morfología- Filogenia } \\
\text { Extinciones }\end{array}$ & $\begin{array}{l}\text { Ritmos evolutivos } \\
\text { Morfología-Filogenia } \\
\text { Extinciones }\end{array}$ & $\begin{array}{l}\text { ooooo } \\
\text { xoooo } \\
\text { xooo }\end{array}$ & $\begin{array}{l}\text { xxxxo } \\
\text { xxooo } \\
\text { xxxoo } \\
\text { xoooo }\end{array}$ \\
\hline BIOEVENTOS & & $\begin{array}{l}\text { Extinciones } \\
\text { Colonizaciones } \\
\text { Ciclos } \\
\text { Fac. anóxicas } \\
\end{array}$ & $\begin{array}{l}\mathrm{xx} \\
\mathrm{xx} \\
\mathrm{xx} \\
\mathrm{xo} \\
\end{array}$ & $\begin{array}{l}\text { xxxxx } \\
\text { xxooo } \\
\text { xoooo } \\
\text { xxooo } \\
\end{array}$ \\
\hline Análisis de cuencas & $\begin{array}{l}\text { Análisis de cuencas } \\
\text { Evolución temporal } \\
\text { Oceanografía }\end{array}$ & Oceanografía & & $\begin{array}{l}\mathrm{xxx} x \mathrm{x} \\
\mathrm{xxo00}\end{array}$ \\
\hline $\begin{array}{l}\text { Microestratigraf. } \\
\text { Ecocstratigrafía } \\
\text { Biofacies }\end{array}$ & & Biofacies & & xxooo \\
\hline $\begin{array}{l}\text { Geoquímica } \\
\text { Isótopos }\end{array}$ & & $\begin{array}{l}\text { Geoquímica orgánica } \\
\text { Isótopos } \\
\text { Pal. molecular }\end{array}$ & $\begin{array}{l}x x \\
x x \\
x+000\end{array}$ & \begin{tabular}{|l|} 
ooooo \\
xooooo \\
xoooo \\
\end{tabular} \\
\hline
\end{tabular}

Tabla 15. Intereses más destacados de los paleontólogos españoles. Obtenido de propuestas en trabajos de prospectiva y de los intereses y carencias detectados. Se hace una valoración del grado de interés (número de símbolos) y de la dedicación al tema en España separando los "Aspectos generales" de carácter teórico del tratamiento para grupos concretos. 
dominen técnicas de interés (geoquímicas, isótopos, etc.). El cuello de botella que suponía carecer de una infraestructura técnica se puede considerar superado.

Una proyectiva realista establecería que los temas más probables para el futuro son los que en el cuadro aparecen como carencias que ya han empezado a tratarse: grupos minoritarios, vertebrados no mamíferos, Tafonomía aplicada, Morfogénesis general y de grupos concretos, Paleoclimatología y Oceanografía, Paleobiogeografía, relación Morfología-Filogenia, extinciones, bioeventos de todo tipo, biofacies, etc.

\section{Otras comunidades científicas, sociedades, etc.}

Tal vez lo que más ha cambiado, y va a cambiar en el futuro, son las demandas de otras ciencias y las respuestas a las mismas. Al tiempo hay que señalar que desde los años 80 hay proyectos multidisciplinares para verificar propuestas paleontológicas, lo que ha roto el carácter de ciencia auxiliar que parecía tener la Paleontología. El resto de la comunidad científica inmediata desconoce el trabajo y los intereses de los paleontólogos, excepto en dos puntos: el bioestratigráfico en relación con la Geología y la búsqueda de grandes principios y leyes en relación con la Biología y la Geología.

Aunque los trabajos de infraestructura geológica son cada vez menos frecuentes, la Bioestratigrafía y la Biocronología se solicitan para la datación de fenómenos geológicos, petrológicos, estratigráficos, etc.. La aplicación de técnicas geofísicas y geoquímicas en rocas sedimentarias necesita cada vez más precisiones bioestratigráficas que permitan las correlaciones. Esto lleva a la Paleontología a:

a) estudio de grupos fósiles de especial interés en terrenos con problemas de datación. Los acritarcos, el nanoplancton, especialmente el no calcáreo, así como la Palinología se utilizan cada vez más.

b) precisión de las escalas bioestratigráficas clásicas para mejorar las correlaciones y establecimiento de escalas bioestratigráficas múltiples.

c) desarrollo de métodos de mayor precisión temporal: tafonómicos, ecoestratigráficos, etc., que permiten apoyar más ampliamente la Estratigrafía de eventos, tanto la secuencial como la relativa a cambios bruscos o catastróficos (Einsele et al, eds., 1991).

Desgraciadamente las demandas de otras ciencias hacia la Paleontología no son claras ni explícitas; desde la Geología se observan con interés los avances en Tafonomía y Bioestratigrafía por su aplicación en análisis de cuencas; desde la Biología los estudios morfológicos y poco más. El desconocimiento del trabajo paleontológico y sus posibilidades es casi total, incluso en centros multidisciplinares donde podría darse una colaboración. Salvo excepciones, no hay demandas desde otros campos como la Ecología marina, Prehistoria, etc.. Son una excepción los investigadores de prestigio que tienen una visión integradora de la ciencia; sirva como ejemplo Margalef (1981). Se desprecia la capacidad de los paleontólogos para realizar la dimensión histórica del medio ambiente. Los historiadores colaboran en el establecimiento de series de acontecimientos ambientales; sin embargo no existe una demanda equivalente hacia la Paleontología del Cuaternario o en cualquier caso hacia la Paleontología por su capacidad para establecer modelos de evolución temporal.
Por otra parte existen demandas claras desde la Paleontología a otras ciencias y esto afecta a los temas de mayor interés para los paleontólogos: estudio integral de yacimientos, análisis de secuencias, Oceanografía, Biogeografía, Paleoecología, cambios en los ritmos evolutivos y en la evolución de las comunidades en relación con cambios climáticos, geológicos, etc.

\section{Demandas de los Centros}

Los centros más antiguos se han desarrollado por la incorporación de investigadores e investigaciones que complementaban las existentes, la mayor parte de las veces ligadas a una región y a un programa geológico. El crecimiento ha seguido, fundamentalmente, criterios bioestratigráficos: tener más investigadores que cubrieran mayor número de grupos y/o más extensión temporal.

En los últimos años, en unos centros más que en otros, se ha producido un cambio en esa tendencia por:

a.- el desarrollo de centros no ligados a estudios de Ciencias Geológicas o a grupos de geólogos.

b.- un crecimiento importante del número de investigadores en los centros existentes

c.- una mayor formación teórica de los estudiantes.

d.- aparición de gran número de centros nuevos con muy escaso número de investigadores.

Esto ha llevado a romper las especializaciones de los centros antiguos que dedican su capacidad de formación a temas más generales y/o de mayor contenido teórico (Morfología funcional, Paleoecología, Paleoclimatología, Tafonomía, etc.), que en parte rentabilizan el material y los conocimientos acumulados. El crecimiento de las Facultades de Ciencias Biológicas y la incorporación de sus licenciados a la Paleontología han desligado a los grupos de investigación de los temas bioestratigráficos. La mejor formación biológica y teórica de los investigadores noveles les permite abordar fácilmente cualquier grupo de organismos fósiles.

\section{DEMANDAS SOCIO-CULTURALES}

Se incluyen en este apartado las demandas docentes universitarias, aunque bien pudieran haberse incluido en el apartado anterior por lo relacionadas que están las comunidades científicas con la Universidad, toda vez que la docencia preuniversitaria es prácticamente inexistente.

Esto último es tanto más grave cuanto que responde a una subversión de los temarios que sí contienen materias paleontológicas aunque en programas poco actualizados y textos con tratamientos lamentables. El panorama se completa con la escasa formación paleontológica del profesorado no universitario procedente de Ciencias Biológicas. Está claro que la mejora en este campo corresponde a la comunidad paleontológica a través de programas de formación continuada y de publicaciones asequibles y adecuadas. Convendría hacer una reflexión seria, paralela a la organizada para la Geología, que intente superar los problemas que Sequeiros \& Martínez Urbano (1992) encuentran en los estudiantes no universitarios.

La enseñanza universitaria en España y en Europa ha sido tratada en extenso por diversos autores desde 1980 
(Babin, 1981, 1992; Moody, 1981, etc.) y casi todos ellos coinciden en: estudiantes con falta de conocimientos paleontológicos básicos, y un rechazo más o menos generalizado de los claustros docentes hacia la enseñanza de esta ciencia; más en los de Biología que en los de Geología.

No hay que hacer una reafirmación del valor científico y formativo de la Paleontología, pues es opinión general que es una candidata idónea a materia reglada (obligatoria u optativa) en las licenciaturas de Ciencias Geológicas, Biológicas, del Mar, Medio Ambiente o en los títulos de Ingenieros Superiores o Técnicos de la rama minera, así como en Arqueología y Prehistoria.

La posición actual de la Paleontología en esos estudios es consecuencia de un proceso en gran medida ajeno a la filosofía pedagógica. En Ciencias Geológicas el mantenimiento de la enseñanza de la Paleontología pasa hoy y para un próximo futuro por:

- peso de la tradición que hace difícil a los universitarios renunciar a las bases de su propia formación.

- casualidades políticas afortunadas que han contrarrestado el peso de los que pedían su supresión o reducción drástica. Su situación como asignatura troncal, con un valor inicial similar al del resto de materias básicas geológicas, es una propuesta política que no se hubiese generado en las Facultades.

- tozudez del sistema de gobierno de las Universidades donde se emplean métodos democráticos para dilucidar problemas científicos. El peso de los paleontólogos, o sus alianzas con quienes compartían rechazo, ha permitido mantener las enseñanzas a un nivel proporcional al de épocas anteriores.

Es duro admitir este análisis, intencionadamente exagerado y simplista, pero al hacerlo encontraremos algunas de las razones de la situación actual de la docencia en la Universidad. Una vez mantenida la troncalidad y en su caso la obligatoriedad en el primer ciclo, no ha resultado difícil conseguir el resto de la oferta ya existente, o incluso aumentarla en el segundo ciclo.

En Ciencias Biológicas las diferencias entre Universidades son grandes y son muchas las que no tienen enseñanzas de Paleontología. No era de esperar apoyo político en este caso ya que la implantación de la asignatura, cuando no existía, suponía un aumento presupuestario. La suerte ha dependido de los claustros; allí donde había una tradición se han mantenido los niveles anteriores, no sin grandes esfuerzos.

Peor suerte ha corrido la Paleontología en las nuevas licenciaturas de Medio Ambiente y Ciencias del Mar. Las enseñanzas casi no existen y las de Geología se han encomendado, por el sistema de automultiplicación, a profesores pertenecientes a las ramas más dispares.

Desde un punto de vista científico-académico hay que estar de acuerdo con Babin (op. cit) que achaca la recesión de la Paleontología en Europa a: su carácter de ciencia de interfase entre la Biología y la Geología; la escasez de empleos de paleontólogos; la imagen, real o no, de la Paleontología como una ciencia trasnochada, enciclopédica, alejada de la experimentación aunque con un halo romántico. Justamente este último aspecto es el que hace que exista una retroalimentación y que la Paleontología tenga aceptación entre los estudiantes de Biología de Sistemas.

Todo lo anterior se debería completar con: ¿Qué Paleontología enseñar?. El tema es tan amplio que se escapa de la intención de este trabajo y queda para la solicitada reflexión sobre la enseñanza de la Paleontología. Si hasta ahora la crítica se ha dirigido a los compañeros de otras ciencias ahora le toca el turno a los paleontólogos. Las enseñanzas no regladas, los cursos, cursillos, etc., que se imparten en la Universidad o por otras iniciativas, se suelen realizar a propuesta del profesorado y, sin duda, en eso estamos por detrás de otras comunidades científicas y de la propia demanda social. Existe un pudor excesivo en sacar nuestra ciencia fuera de las aulas y de los circuitos habituales y no parece coherente que de los miles de cursos y cursillos que anualmente se realizan, no alcancemos ni el 0,5\% de los mismos. Superar esto permitirá abrir la Paleontología a la sociedad.

Su carácter de ciencia frontera, y su gran capacidad formativa la hacen idónea para promocionarla como estudios de libre elección de los estudiantes. Esto se ve reforzado por:

- masificación de la Universidad que lleva, o debe llevar, a los estudiantes a escoger la formación general frente a la especializada

- entrada cada vez mayor, en casos hasta el 20\% (datos de la Universidad de Granada), de estudiantes de segunda oportunidad, mayores de 28 años, que buscan en los estudios una satisfacción cultural más que profesional y un título universitario que promocione su experiencia profesional.

- mayor incidencia de las enseñanzas no regladas en el sistema que pueden llegar, en un cálculo no optimista, a suponer el $30 \%$ del esfuerzo docente de la Universidad (Rivas, 1993) y muy superior si se tienen en cuenta los sistemas de formación contínua (Teichler, 1990; Otala, 1992).

La creación de actividades de carácter más abierto, formativas y divulgativas, debería ser una de las actividades de la Sociedad Española de Paleontología; actividad no tanto para satisfacer, como ahora, a la propia comunidad científica como para promover la Paleontología en la sociedad. Artículos de opinión y divulgaciones en los medios de comunicación cambiarían el signo de nuestra presencia en los mismos.

La museística, en alza en estos momentos, supone una esperanza; se han recuperado y/o puesto en marcha nuevos centros museísticos: Sevilla, Córdoba, Orce, Valencia, Zaragoza, Barcelona. Hay una propuesta firme para crear otro en Burgos y existe sensibilidad social para iniciar el trabajo en otros puntos. En cualquier caso la diversificación de los riesgos es una buena política. A este futuro contribuyen los numerosos centros con paleontólogos de plantilla, que por la inercia de la administración no pueden mas que mantenerse o crecer: Coruña, Extremadura, Córdoba, Jaén, Cantabria, Cádiz, etc.

En otro orden de cosas, el futuro editorial es prometedor. Han aparecido nuevos libros de Paleontología, se editan monografías sobre temas de interés para la sociedad, y las traducciones son cada vez más frecuentes, aunque la política ministerial no favorece esto, pues el esfuerzo y el tiempo de los investigadores no tienen una recompensa correlativa a la de otras actividades. El presente es, sin duda, mucho más diverso en cuanto a revistas; se han consolidado las nuevas y mejorado o conservado las anteriores, excepto las muy locales que tienden a desaparecer, a la vez que otras del mismo carácter aparecen. La crisis de la Empresa Adaro 
hace peligrar la continuidad de la Revista Española de Micropaleontología, sin duda la española de más prestigio en el sector y la más internacional. Su pérdida sería algo irreparable que ni las instituciones ni esta Sociedad pueden permitirse. Existen fórmulas que pueden asegurar el futuro que ahora parece incierto. La Revista Española de Paleontología tiene un gran nivel y atrae a investigadores extranjeros en un número impensable hace unos años. Esta mejora, importante, no es suficiente y aunque ha aumentado la calidad de los trabajos publicados a través de la crítica por pares, no se ha definido una política editorial que podría prestigiarla más.

\section{DEMANDAS SOCIO-ECONÓMICAS}

No son previsibles demandas desde el sector productivo; es más, las que había están desapareciendo paralelamente a la crisis de la Geología. En los centros públicos existe una comunidad que puede dar respuesta, por preparación y capacidad legal, a las demandas sociales, lo que traerá consigo la práctica desaparición del paleontólogo profesional en las empresas.

Las demandas de trabajo en temas medioambientales han llevado a que se formen equipos multidisciplinares dedicados, en especial, al estudio del impacto y la restitución ambiental y, en menor proporción, al tratamiento de residuos sólidos y líquidos. Se ha abandonado para equipos técnicos específicos el tratamiento de la contaminación industrial. Buena parte de los geólogos y de las empresas de Geología se han reconvertido a esta demanda y en ella el paleontólogo puede encontrar un futuro, incluso con ventaja frente a los nuevos licenciados en Medio Ambiente.

El interés por estos temas y por los cambios ambientales seculares ha ido acercando a los paleontólogos españoles a estos estudios y a ello no es ajeno el interés creciente por el Neógeno-Cuaternario con enfoques las más de las veces paleoecológicos. Paralelamente, se ha mejorado la capacidad de formación y eso debe redundar en una oferta mucho más decidida en los aspectos ambientales.

Para finalizar dos consideraciones:

- la primera oída en 1987 al Dr. Rosell. El estudio de algunos medios marinos, en especial de los más profundos, sólo es accesible de forma fiable a través de los yacimientos geológicos, que nos los ponen al alcance de la mano. Debemos interesarnos por ese campo que se nos reserva casi en exclusiva y establecer modelos a partir de lo ocurrido entre el Paleozoico y el Neógeno.

- la segunda, sentida desde hace años, formulada por la Dra. Linares en 1993 y recogida en una publicación del Museo de Leiden. Hasta ahora la Paleontología y, en general, la Geología, acudía a los modelos actuales para interpretar el pasado. La metodología paradigmática nos permite abordar directamente problemas del pasado, para hacer predicciones sobre el futuro, que es lo que más demanda la sociedad actual. El pasado y su estudio son las claves para predecir el futuro.

\section{AGRADECIMIENTOS}

Mi agradecimiento al comité organizador por la invitación para dar esta conferencia; de M. ${ }^{a}$ José Comas y Sergio Rodríguez sabía de su amistad, no de su confianza. A mis colegas que desinteresadamente han suministrado datos actualizados para poder conocer las comunidades científicas de los distintos centros. Finalmente al Prof. García Alcalde que ha hecho una lectura crítica del texto original y ha ayudado a transformar lo que era para "decir" en algo para "leer".

El momento de corrección de pruebas coincide con la muerte de D. José Ramírez del Pozo, que afecta a toda la comunidad paleontológica y hace desaparecer al amigo y al científico, a la vez que a uno de los centros que se contabilizan en este trabajo.

\section{BIBLIOGRAFÍA}

Aguirre, E. (coord.) 1989. Paleontología. Nuevas tendencias. Ed. C.S.I.C. Madrid, 433 pp.

Alberch, P. 1991. Del gen al fenotipo: sistemas dinámicos y evolución morfológica. In: El estudio de la Forma Orgánica y sus consecuencias en Paleontología sistemática, Paleoecología y Paleontología evolutiva. Rev. Esp. Paleont. nº Extr., 13-19

Allison, P. A y Briggs, D. E. G. (Eds.) 1991. Taphonomy. Realeasing the Data Locked in the Fossil Record. Plenum Press, New York, Londres, $\mathrm{x}+560 \mathrm{pp}$.

Álvarez, F. y Conway Morris, S. (Ed.) 1992. Palaeobiology: Preparing for the Twenty-First Century. Centro Reuniones Internacionáles sobre Biología. Instituto Juan March de Estudios e Investigaciones, Madrid. $140 \mathrm{pp}$.

Babin, C. 1981. ¿Qué Paleontología conviene enseñar?. In: Concept and Method in Palaeontology (Ed. Martinell, J.). Acta Geol. Hispánica, 16, 95-102.

Babin, C. 1992. La enseñanza de la Paleontología en Europa, de ayer a mañana. In: Paleontología y Sociedad. Actas de las VI Jornadas de Paleontología. Sociedad Española de Paleontología. \& Dpto. Estratigrafía y Paleontología, Univ. Granada, Granada, 7-18.

Brett, C. E. y Speyer, S. E. 1990. Taphofacies. In: Palaeobiology. A Synthesis (Eds. D. E. G. Briggs \& P. R. Crowther). Blackwell. Sc. Publications, Oxford, 258-263.

Briggs, D. E. G. y Crowther, P. R. (Eds.) 1990. Palaebiology. A Synthesis. Blackwell Sc. Publications, Oxford, 583 pp.

Einsele, G., Ricken, W. y Seilacher, A. (Eds.) 1991. Cycles and Events in Stratigraphy. Springer-Verlag, Berlin, Heidelberg, N. York., 955 pp.

Eldredge, N. 1989. Macroevolutionary dynamics. Species, Niches \& Adaptive peaks. McGraw-Hill Publising Company, New York, $\mathrm{xi}+226 \mathrm{pp}$.

Fernández López, S. 1991. Sistemas tafonómicos: función y evolución. In: El estudio de la Forma Orgánica y sus consecuencias en Paleontología sistemática, Paleoecología y Paleontología evolutiva. Rev. Esp. Paleont., $\mathbf{n}^{\circ}$ Extr., 21-34.

Fernández López, S. (Ed.) 1993. Conferencias de la reunión de Tafonomía y Fosilización. Ed. Univ. Complutense, Madrid, 329 pp. 
Gould, S. J. 1977. Eternal Metaphors of Palaeontology. In: Patterns of evolution (Ed. A. Hallam). Elsevier Sc. Publising Company, Amsterdam, 1-26.

Gregory, J. T., Bacskai, J. A., Shkurkin, G. V. y Bryant, L. A. (Eds.) 1983. Bibliography of Fossil Vertebrata 1980. American Geological Institute, Falls Church, viii +471 pp.

Gregory, J. T., Bacskai, J. A., Shkurkin, G. V., Winans, M. C. y Rauscher,B. H. (Eds.), 1988. Bibliography of Fossil Vertebrata 1985. Society of Vertebrate Paleontology, Los Angeles, viii +568 pp.

Gregory, J. T., Bacskai, J. A., Shkurkin, G. V., Winans, M. C. y Rauscher, B. H. (Eds.) 1989a; 1989b; 1991; 1992. Bibliography of Fossil Vertebrata 1986; 1987; 1988; 1989. Society of Vertebrate Paleontology, Lincoln, ix +509 pp.; viii +472 pp.; xiii + 481 pp.; xiii +495 .

Gregory, J. T., Bacskaia, J. A., Shkurkin, G. V. y Rauscher, B. H. (Eds.), 1993. Bibliography of Fossil Vertebrata 1990. Society of Vertebrate Paleontology, Lincoln, xiii +455.

Jablonski, D. 1986. Causes and consecuences of Mass Extintions: a comparative approach. In: Dynamics of Extintion (Ed. D.K. Elliot). John Wiley, Londres, 183-217.

Hoffman, A. 1988. Arguments on Evolution. A paleontologists perspective. Oxford Univ. Press, N. York, Oxford, 274 pp.

Hoffman, A. 1990. The Past Decade and the Future. In: Palaeobiology a Synthesis (Eds. D. E. G. Briggs \& P. R. Crowther). Blackwell Sc. Publ, Londres, 550-555.

Kauffman, E. G. y Wallister, O. H. (Eds.) 1990. Extintions events in Earth history. Lecture Notes in Earth Sciences, 30, SpringerVerlag, Berlin, Heidelberg, N. York, 432 pp.

Kidwell, S. M. 1991. The Stratigraphy of shell concentrations. In: Taphonomy. Releasing the Data Locked in the Fossil Record (Eds. P. A. Allison \& D. E. G. Briggs). Plennun Press New York, Londres 211-290.

Lamolda, M. A.; Kauffman, E. G. y Wallister, O. H. (Eds.) 1988. Palaeontology and Evolution: Extintion events. III Jornadas de Paleontología, Bilbao. Rev. Esp. Paleont. $\mathbf{N}^{0}$ Extr. 155 pp.

Linares, A. 1993. Hitos en el desarrollo de la Paleontología en Andalucía desde mediados del siglo XIX. In: Comunicaciones IX Jornadas de Paleontología (Ed. J.M. González-Donoso). Málaga, i-xv.

López Martínez, N. y Truyols, J. 1994. Paleontología. Conceptos y Métodos. Ed. Síntesis, Madrid, 334 pp.

Margalef, R. 1981. Asimetrías introducidas por la operación de la energía externa en secuencias de sedimentos y poblaciones. In: Concept and Method in Palaeontology (Ed. J. Martinell). Acta Geol. Hispánica, 16, 35-38.

Martinell, J. (Ed.) 1981. Concept and Method in Paleontology. Acta Geol. Hispánica, 16, 114 pp.
Molina, E. 1982. Introducción de la Micropaleontología en España. In: Actas del II Congreso Soc. Española de Historia de la Ciencia (Ed. M. Hormigón). Jaca, 250-261.

Moody, R. T. J. 1981. To teach or no to teach. Toward the development of a conceptual and technique biased syllabus in Palaeontology. In: Concept and Method in Paleontology (Ed. J. Martinell). Acta Geol. Hispa., 16, 89-94.

Morales, J. y Rivas, P. 1987. Ponencia: Bioestratigrafía. In: Prospectiva en Ciencias de la Tierra, (Ed. J. A. Vera). C.S.I.C., Madrid, 99-108.

Otala, L. 1992. European Approaches to Lifelong Learning. The European University-Industry Forum, Ginebra, 112 pp.

Ramírez del Pozo, J. 1992. La Micropaleontología en España: situación actual y perspectivas. In: Paleontología y Sociedad. Actas de las VI Jornadas de Paleontología. Sociedad Española de Paleontología. \& Dpto. Estratigrafía y Paleontología, Univ. Granada, Granada, 19-36.

Raup, D. V. 1990. Extintion: bad genes or bad luck?. W. W. Norton, N. York, 210 pp.

Rivas, P. 1993. Intervenciones académicas. Serv. Publi. Univ. Granada, Granada, 189 pp.

Runnegar, B. y Schopf, J. W. (Con.) 1988. Molecular evolution and the fossil record. Short Courses in Paleontology, 1, $167 \mathrm{pp}$.

Sepkoski, J. J. 1978. A kinetic model of Phanerozoic taxonomic diversity. I. Analysis of marine orders. Paleobiology, 4, 223-251.

Seilacher, A. 1990. Taphonomy of Fossil-Lagerstätten. Overwiew. In: Palaeobiology. A Synthesis (Eds. D. E. G. Briggs \& P. R. Crowther). Blackwell Sc. Publications, London, 266-270.

Sequeiros, L. y Martínez Urbano, M. 1992. Ideas previas de los alumnos no universitarios sobre la Paleontología. In: Paleontología y Sociedad. Ed. Sociedad Española de Paleontología y Dpto. de Estratigrafía y Paleontología, Univ. Granada, Granada, 183-197.

Teichler, U. 1990. The challenge of lifelong learning for the university. In: University competence and industry. Creaction, 92-4, 53-68.

Truyols, J. 1988. Desarrollo histórico de la Paleontología contemporánea en España. In: Historia de la Paleontología. Real Acd. Cienc. Exact. Físic. Natural, Madrid, 45-67.

Vera, J. A. (ed.), 1986. Prospectiva en Ciencias de la Tierra. C.S.I.C., Madrid, 194 pp.

Manuscrito recibido: 16 de diciembre, 1994 Manuscrito aceptado: 16 de diciembre, 1994 